Received: 06.10.2021

Revised: 06.12.2021

Accepted: 24.12 .2021

DOI: $10.17804 / 2410-9908.2021 .6 .006-018$

\title{
SHORT-RANGE ORDER IN Fe-Cr ALLOYS IRRADIATED WITH ELECTRONS AT LOW TEMPERATURES AND Cr SOLUBILITY IN $\alpha$-IRON NEAR $270 \mathrm{~K}$
}

\author{
A. L. Nikolaev \\ M.N. Miheev Institute of Metal Physics, Ural Branch of the Russian Academy of Sciences, \\ 18 S. Kovalevskoy St., Ekaterinburg, 620108, Russian Federation \\ iD https://orcid.org/0000-0002-0858-9820 \nikolaev@imp.uran.ru \\ Corresponding author. E-mail: nikolaev@imp.uran.ru \\ Address for correspondence: ul. S. Kovalevskoy, 18, Ekaterinburg, 620108, Russian Federation \\ Tel.: +7 (343) 3783850
}

\begin{abstract}
Chromium has limited solubility in $\alpha$-iron. It is shown that unsaturated $\mathrm{Fe}-\mathrm{Cr}$ alloys should demonstrate short-range ordering (i.e. tendency to neighboring of $\mathrm{Fe}$ and $\mathrm{Cr}$ atoms). The type of shortrange ordering in several $\mathrm{Fe}-\mathrm{Cr}$ alloys is revealed from the data on the recovery of residual electrical resistivity after low temperature electron irradiation at sufficiently low temperatures. The combined consideration of the data on short-range ordering and chromium solubility at $570 \mathrm{~K}$ (Scr. Mater. $2014 \mathrm{v}$. 74 , p. 48 and ibid 2016 v. 122, p. 31) gives the confidence interval for the solubility limit near $270 \mathrm{~K}$ equal to $8.8 \pm 0.4$ at. $\% \mathrm{Cr}$.
\end{abstract}

Keywords: $\mathrm{Fe}-\mathrm{Cr}$ alloys, $\mathrm{Cr}$ solubility limit in $\alpha$-iron, electron irradiation, recovery of residual electrical resistivity, short-range ordering.

\section{Acknowledgment}

The research was carried out under a state assignment from the Ministry of Science and Higher Education of the Russian Federation (theme Function, No. AAAA-A19-119012990095-0).

\section{References}

1. Bachhav Mukesh, Odette G. Robert, Marquis Emmanuelle A. $\alpha^{\prime}$ precipitation in neutron-irradiated $\mathrm{Fe}-\mathrm{Cr}$ alloys. Scripta Materialia, 2014, vol. 74, pp. 48-51. DOI: $10.1016 /$ j.scriptamat.2013.10.001.

2. Tissot O., Pareige C., Meslin E., Decamps B., Henry J. Kinetics of $\alpha^{\prime}$ precipitation in an electron-irradiated Fe15Cr alloy. Scripta Materialia, 2016, vol. 122, pp. 31-35. DOI: 10.1016/J.SCRIPTAMAT.2016.05.021.

3. Mirebeau I., Hennion M., Parette G. First measurement of short-range-order inversion as a function of concentration in a transition alloy. Phys. Rev. Letters, 1984, vol. 53, iss. 7, pp. 687-690. DOI: 10.1103/PHYSREVLETT.53.687.

4. $\quad$ Filippova N.P., Shabashov V.A., Nikolaev A.L. Mössbauer Study of Irradiation-Accelerated Short-Range Ordering in Binary Fe-Cr Alloys. Physics of Metals and Metallography, 2000, vol. 90, No. 2, pp. 145-152.

5. Dimitrov C., Dimitrov O. Composition dependence of defect properties in electronirradiated Fe-Cr-Ni solid solutions. J. Phys. F: Met. Phys., 1984, vol. 14, pp. 793-811. DOI: $10.1088 / 0305-4608 / 14 / 4 / 005$. 
6. Vaessen P., Lengeler B., Shilling W. Recovery of electrical resistivity in electronirradiated concentrated silver-zinc alloys. Rad. Effects, 1984, vol. 81, pp. 277-292. DOI: 10.1080/00337578408206075.

7. Ruban A.V., Korzhavyi P.A., Johansson B. First-principles theory of magnetically driven anomalous ordering in bcc Fe-Cr alloys. Phys. Rev. B, 2008, vol. 77. iss 9, pp. 094436. DOI: 10.1103/PHYSREVB.77.094436.

8. Kohl W., Scheffel R., Heidsiek H., Lucke K. Investigation of the kinetics of short-range order formation and quenched-in vacancy annihilation in Au-15 at. \% Ag by resistivity measurements. Acta metall., 1983, vol. 31, No. 11, pp. 1895-1908. DOI: 10.1016/0001-6160(83)90135-9.

9. Nikolaev A.L. Specificity of stage III in electron-irradiated Fe-Cr alloys. Phil. Mag., 2007, vol. 87, No. 31, pp. 4847-4874. DOI: 10.1080/14786430701468977.

10. Nikolaev A. L. Difference approach to the analysis of resistivity recovery data for irradiated short-range ordered alloys. Phil. Mag., 2009, vol. 89, No. 12, pp. 1017-1033. DOI: 10.1080/14786430902835651.

11. Nikolaev A. L. Recovery of electrical resistivity, short-range order formation and migration of defects in electron-irradiated Fe-4Cr alloy doped with carbon. Phil. Mag., 2011, vol. 91, No. 6, pp. 879-898. DOI: 10.1080/14786435.2010.534740.

12. Maury F., Lucasson P., Lucasson A., Faudot F., Bigot J. A study of irradiated FeCr alloys: deviations from Matthiessen's rule and interstitial migration. J. Phys. F: Met. Phys., 1987, vol. 17, pp. 1143-1165. DOI: 10.1088/0305-4608/17/5/014.

13. Nikolaev A.L. Stage I of recovery in $5 \mathrm{MeV}$ electron-irradiated iron and iron-chromium alloys: the effect of small cascades, migration of di-interstitials and mixed dumbbells. $J$. Phys.: Condens. Matter., 1999, vol. 11 (44), pp. 8933-8644. DOI: 10.1088/0953-8984/11/44/302. 
Подана в журнал: 06.10.2021

УДК 539.12.043:620.192.63

DOI: $10.17804 / 2410-9908.2021 .6 .006-018$

\title{
БЛИЖНИЙ ПОРЯДОК В ОБЛУЧЕННЫХ ЭЛЕКТРОНАМИ ПРИ НИЗКИХ ТЕМПЕРАТУРАХ СПЛАВАХ Ғе-СР И РАСТВОРИМОСТИ ХРОМА В $\alpha$-ЖЕЛЕЗЕ ВБЛИЗИ 270 К
}

\author{
А. Л. Николаев \\ Институт физики металлов Уральского отделения Российской академии наук, имени М.Н.Михеева, \\ д. 18, ул. С.Ковалевской, Екатеринбург, Российская Федераџия \\ (iD https://orcid.org/0000-0002-0858-9820 nikolaev@imp.uran.ru \\ *Ответственный автор. Электронная почта: nikolaev@imp.uran.ru \\ Адрес для переписки: ул. С. Ковалевской, 18, Екатеринбург, Российская Федерация \\ Тел.: +7 (343) 378-38-50
}

Хром имеет ограниченную растворимость в $\alpha$-железе. Ненасыщенные сплавы $\mathrm{Fe}-\mathrm{Cr}$ должны демонстрировать ближнее упорядочение (БУ) по типу порядка. Из данных по возврату остаточного электросопротивления после низкотемпературного облучения электронами высоких энергий установлен тип БУ в ряде сплавов $\mathrm{Fe}-\mathrm{Cr}$ при достаточно низких температурах. Совместное рассмотрение данных по БУ и пределу растворимости Сr при $570 \mathrm{~K}$ (Scr. Mater. 2014 v. 74, p. 48 и ibid 2016 v. 122, p. 31) позволяют установить доверительный интервал для предела растворимости вблизи 270 К, равный 8,8土0,4 ат. \% Cr.

Ключевые слова: сплавы $\mathrm{Fe}-\mathrm{Cr}$, предел растворимости $\mathrm{Cr}$ в $\alpha$-железе, облучение электронами, возврат остаточного электросопротивления, ближнее упорядочение.

\section{1. Введение}

Богатые по железу сплавы Fe-Cr являются основой для множества ферритных и ферритно-мартенситных нержавеющих сталей, которые широко используются в промышленности, включая применение в ядерных реакторах благодаря хорошим механическим свойствам, химической стойкости и высокой устойчивости к вакансионному распуханию. Растворимость хрома в $\alpha$-железе является ограниченной. Из-за этого ограничения высокохромистые (выше 17-18 ат. \% Сr) сплавы и стали имеют тенденцию к расслоению на богатые по железу ( $\alpha$-фаза) и хрому ( $\alpha^{\prime}$-фаза) компоненты, что приводит к охрупчиванию. Это охрупчивание впервые наблюдалось при старении при $475{ }^{\circ} \mathrm{C}$ и известно как «хрупкость при $475{ }^{\circ} \mathrm{C».} \mathrm{Это}$ явление серьезно ухудшает механические свойства высокохромистых сплавов и ферритных сталей. Однако это ухудшение может происходить и в сплавах, и сталях с более низким содержанием хрома ( 12-13 ат. \% Cr), и при более низких температурах при их использовании в ядерных реакторах, где миграция неравновесных дефектов, возникающих при облучении нейтронами, ускоряет процессы диффузии и фазовых превращений при температурах, существенно ниже $750 \mathrm{~K}\left(475^{\circ} \mathrm{C}\right)$, и концентрациях хрома, близких к пределу растворимости.

Чтобы понимать поведение расслаивающихся сплавов, необходимо знать предел растворимости хрома $C^{s}$, включая его значения при достаточно низких температурах. В последние годы в ряде работ была экспериментально определена растворимость хрома в $\alpha$ железе вблизи 570 K [1, 2]. В этих работах пересыщенный твердый раствор сплавов $\mathrm{Fe}-\mathrm{Cr}$ доводили практически до полного распада и равновесную концентрацию хрома в обедненной матрице (предел растворимости) измеряли с помощью атомно-зондовой томографии. Атомная подвижность вблизи 570 К была чрезвычайно низкой и чтобы ускорить ее с помо- 
щью миграции неравновесных дефектов, применяли облучение нейтронами и электронами высоких энергий.

Однако при температурах, существенно ниже 570 К, рекомбинация неравновесных дефектов увеличивается на много порядков и способность облучения ускорять распад твердого раствора резко падает. Поэтому подход $[1,2]$ реализовать не удастся. Тем не менее, даже при температурах существенно ниже 570 К мигрирующие радиационные дефекты способны ускорять процессы ближнего упорядочения (БУ). В системе $\mathrm{Fe}-\mathrm{Cr}$ существует два типа БУ [3] - БУ по типу порядка (далее просто ближний порядок - БП) и БУ по типу расслоения (далее просто ближнее расслоение - БР). При низких концентрациях хрома наблюдается БП, а при концентрации чуть ниже 10 ат. \% $\mathrm{Cr}\left(C_{C r}^{\text {inv }}\right)$ происходит переход к БР, т. е. имеет место инверсия типа БУ. Этот концентрационный переход наблюдали как при термических отжигах в интервале 700-900 К [3], так и после облучения электронами при 370-420 К [4].

Очевидно, что образование обогащенных по хрому кластеров (зародышей $\alpha^{\prime}$-фазы) возможно только при условии, что соседство какого-либо атома $\mathrm{Cr}$ с другими атомами $\mathrm{Cr}$ является энергетически выгодным. С точки зрения БУ это означает, что в распадающихся (пересыщенных) сплавах должно происходить БР. Отсюда следует, что при концентрациях хрома ниже $C_{C r}^{i n v}$ твердый раствор является устойчивым относительно расслоения на $\alpha-$ и $\alpha^{\prime}$-фазы, так как стремление атомов хрома соседствовать с атомами железа (что происходит при БП) не совместимо с зарождением обогащенных по хрому кластеров (зародышей $\alpha^{\prime}-$ фазы). Поэтому $C_{C r}^{\text {inv }}$ является либо пределом растворимости, либо по крайней мере его нижней оценкой, т. е. $C_{C r}^{\text {inv }} \leq C^{s}$.

В работе [3] показано, что процессы БУ разного типа по-разному влияют на изменение остаточного электрического сопротивления (ОЭС): БП увеличивает ОЭС, а БР уменьшает ОЭС. Это дает возможность определять тип БУ с помощью измерений ОЭС.

Хорошо известно, что возврат ОЭС в концентрированных сплавах в процессе ступенчатого изохронного отжига после облучения частицами высоких энергий при низких температурах сопровождается БУ, ускоренным дальней миграцией радиационных дефектов $[5,6]$. Это приводит к появлению в возврате ОЭС соответствующего вклада, вызванного БУ, $\Delta \rho^{S R O}(T)^{1}$.

В сплавах $\mathrm{Fe}-\mathrm{Cr} \Delta \rho^{S R O}(T)$ ведет себя по-разному в зависимости от типа БУ: в случае БП $\Delta \rho^{S R O}(T)$ является положительным и увеличивается с ростом температуры отжига, а в случае БР $\Delta \rho^{S R O}(T)$ является отрицательным и уменьшается с ростом температуры отжига. Поэтому информация как о протекании БУ, так и об его типе может быть получена из данных по возврату ОЭС. Соответственно, если $\Delta \rho^{S R O}>0$, то исследуемый твердый раствор однозначно является ненасыщенным, т. е. концентрация хрома в этом случае ниже $C^{s}$. В противоположном случае $\left(\Delta \rho^{S R O}<0\right)$ никаких однозначных выводов не может быть сделано, так как наличие БР является необходимым, но не достаточным условием расслоения на $\alpha$ - и $\alpha^{\prime}$-фазы. Поэтому из данных по возврату ОЭС можно получить только нижнюю оценку предела растворимости $C_{L}^{s}$, т. е. $C_{L}^{s}<C_{C r}^{i n v} \leq C^{s}$.

Формально значения концентрации хрома, полученные в работах $[1,2]$ для обедненной матрицы $(8,9 \pm 0,2$ и $8,7 \pm 0,5$ at. \%) после длительного старения при 570 К, соответствуют пересыщенному твердому раствору и являются верхней оценкой предела растворимости $C_{H}^{S}(570 \mathrm{~K})$ (хотя и очень близкой к истинному значению последнего при 570 К), так как до-

\footnotetext{
${ }^{1}$ Индекс «SRO» от англ. short-range order; более подробно $\Delta \rho^{S R O}(T)$ обсуждается далее в разд. 2.
} 
стижение точной равновесной концентрации при старении требует бесконечного времени. Это означает, что и при более низких температурах величина $C_{H}^{S}(570 \mathrm{~K})$ также соответствует пересыщенному твердому раствору и является верхней оценкой предела растворимости.

Сопоставление значений $C_{L}^{s}$ и $C_{H}^{S}(570 \mathrm{~K})$ дает доверительный интервал для значений предела растворимости $C^{s}$ при температурах, где возможно определение типа БУ по данным возврата ОЭС, т. е. $C_{L}^{S}<C^{s}<C_{H}^{S}(570 \mathrm{~K})$. $C^{s}$ может только уменьшаться с понижением температуры, и температурная зависимость $C^{s}(T)$ определяет минимальную ширину доверительного интервала при температуре $T$, равную $C_{H}^{S}(570 \mathrm{~K})-C^{S}(T)$. Из теоретических соображений [7] следует, что температурная зависимость $C^{s}(T)$ должна быть слабой. Поэтому ширина доверительного интервала (величина погрешности определения $C^{s}$ ) будет, в основном, определяться разностью $\left(C_{L}^{s}-C^{s}\right)$ примерно равной $C_{L}^{S}-C_{H}^{S}(570 \mathrm{~K})$. Таким образом, задачу по определению предела растворимости с разумной погрешностью можно свести к определению типа БУ в сплаве с концентрацией хрома, немного ниже значений, найденных для $C_{H}^{S}(570 \mathrm{~K})$ в работах [1-2]. Решение именно такой задачи является целью данной работы. Однако оказалось, что указанная задача для подобного сплава просто не решается. Поэтому в работе дополнительно исследовали БУ еще в трех сплавах $\mathrm{Fe}-\mathrm{Cr}$, чтобы понять, как можно определить тип БУ в интересующем нас сплаве.

\section{2. Миграция неравновесных дефектов, БУ и изменение ОЭС в концентрированных сплавах}

2.1. Возврат ОЭС и БУ в концентрированных сплавах при отжиге после облучения электронами при низких температурах

При низких температурах дефекты неподвижны. Поэтому облучение электронами при низких температурах приводит к накоплению дефектов в виде пар Френкеля, состоящих из вакансии и собственного междоузельного атома (CMA). Накопление дефектов (пар Френкеля) приводит к росту ОЭС, а их концентрация определяет величину исходного прироста ОЭС $\Delta \rho_{0}^{d}$, где индекс « $d »$ означает, что прирост обусловлен дефектами. Прирост определяется из соотношения $\Delta \rho_{0}^{d}=\rho_{i r r}-\rho_{0}$, где $\rho_{0}$ и $\rho_{i r r}$ значения ОЭС сплава до и после облучения соответственно.

Стандартная процедура исследования возврата ОЭС предполагает, что облученные образцы подвергают ступенчатому изохронному отжигу, т.е. в процессе отжига ступенчато повышают температуру отжига с шагом $\Delta T$ и выдерживают на каждой ступени одинаковое время $\Delta t$. После отжига на каждой ступени образец погружают в жидкий гелий для измерения ОЭС и определяют текущий прирост ОЭС после отжига при температуре $T$, $\Delta \rho(T)=\rho(T)-\rho_{0}$, где $\rho(T)$ - текущее значение ОЭС после отжига при температуре $T$. Допускается изменение шага отжига, но скорость нагрева $\Delta T / \Delta t$ должна оставаться постоянной.

По результатам ступенчатого отжига получают температурную зависимость восстановления ОЭС $R(T)=\Delta \rho(T) / \Delta \rho_{0}^{d}$. Величину $R(T)$ часто называют возвратом ОЭС. Если $\Delta \rho(T)$ целиком обусловлено присутствием дефектов, то $R(T)$ указывает на долю выживших дефектов после отжига при температуре $T$.

При повышении температуры после облучения дефекты приобретают некоторую подвижность, степень которой определяется величиной температуры, и начинают взаимодействовать друг с другом. Первыми начинаются процессы так называемой ближней миграции, которая приводит к взаимной рекомбинации вакансий и СМА из одной и той же пары Френ-

Nikolaev A. L. Short-range ordering in $\mathrm{Fe}-\mathrm{Cr}$ alloys irradiated with electrons at low temperatures and $\mathrm{Cr}$ solubility in $\alpha$-iron near $270 \mathrm{~K} / /$ Diagnostics, Resource and Mechanics of materials and structures. - 2021. - Iss. 6. - P. 6-18. - 
келя. Нам эти процессы не интересны, так как они никак не влияют на БУ и приводят к простому уменьшению концентрации дефектов и $R(T)$.

При более высоких температурах начинается дальняя миграция дефектов, то есть миграция на расстояния порядка и больше расстояния между отдельными парами Френкеля, и начинается взаимодействие между дефектами из разных пар Френкеля. При этих температуpax миграция дефектов начинает влиять на процессы БУ. БУ является диффузионноконтролируемым явлением, то есть для его протекания требуется диффузионное перемешивание твердого раствора. Степень перемешивания определяется общим числом прыжков всех подвижных дефектов, приходящихся на один атом. Заметное влияние БУ на ОЭС начинается тогда, когда число прыжков дефектов приближается к величине около 1 прыжка/атом [8]. Именно такое число прыжков дефектов набирается при отжигах в начале дальней миграции. При ближней миграции, общее число прыжков дефектов всегда много меньше единицы.

В результате дальней миграции начинаются процессы БУ, которые изменяют ОЭС самого сплава (без учета вклада от дефектов), т. е. $\rho_{0}(T)=\rho_{0}+\Delta \rho^{S R O}(T)$, где $\rho_{0}(T)$ - значение ОЭС самого сплава после отжига при температуре $T$, а $\Delta \rho^{S R O}(T)$ - изменение ОЭС, обусловленное БУ. В силу традиций, изменение ОЭС сплава при отжиге после облучения не рассматривается, а поправка $\Delta \rho^{S R O}(T)$ включается в текущий прирост ОЭС $\Delta \rho(T)$. В результате, $\Delta \rho(T)$ оказывается состоящим из двух компонентов (вкладов), один из которых обусловлен присутствием дефектов $\left(\Delta \rho^{d}(T)\right)$, а второй является результатом БУ $\left(\Delta \rho^{S R O}(T)\right)$, т.е. $\Delta \rho(T)=\Delta \rho^{d}(T)+\Delta \rho^{S R O}(T)$. Соответственно, возврат ОЭС записывается как $R(T)=\Delta \rho^{d}(T) / \Delta \rho_{0}^{d}+\Delta \rho^{S R O}(T) / \Delta \rho_{0}^{d}=R^{d}(T)+R^{S R O}(T)$ и также состоит из двух вкладов (компонентов), обусловленных дефектами и БУ. Нужно иметь в виду, что $\Delta \rho^{d}(T)$ всегда является положительной величиной, а $\Delta \rho^{S R O}(T)$ может быть как положительной, так и отрицательной.

Из сказанного выше следует, что для решения задачи, поставленной во введении, необходимо решить две следующих проблемы: а) как определить температуру $\left(T^{S R O}\right)$, выше которой в возврате $R(T)$ появляется компонента $R^{S R O}(T)$; б) как при температурах выше $T^{S R O}$ определить знак компоненты $R^{S R O}(T)$. В следующем разделе мы покажем, что упомянутые проблемы решаются путем сравнения кривых $R(T)$, полученных в подобных образцах с различной исходной концентрацией дефектов или, другими словами, с различными приростами ОЭС $\Delta \rho_{0}^{d}$. В свете сказанного выше, очевидно, что ниже $T^{S R O}$ определить какие-либо характеристики БУ и, соответственно, растворимость хрома не представляется возможным.

\section{деректов}

2.2 Сравнение возврата ОЭС в подобных образцах с различной конщентрацией

Известно [8], что $\Delta \rho^{S R O}$ является функцией числа прыжков всех подвижных дефектов $N^{d 2}$. Изменение $\Delta \rho^{S R O}(T)$ при отжиге на каждой $i$-й ступени (при температуре $T_{i}$ ) равное $\Delta \rho^{S R O}\left(T_{i}\right)-\Delta \rho^{S R O}\left(T_{i-1}\right)$, где $\Delta \rho^{S R O}\left(T_{i}\right)$ значение $\Delta \rho^{S R O}(T)$ после отжига при температуре $T_{i}-$ мало и с хорошей точностью прямо пропорционально $N^{d}\left(T_{i}\right)$, т. е. числу прыжков дефектов, совершенных при отжиге на данной ступени при $T_{i}$. При нормировке $\Delta \rho^{S R O}\left(T_{i}\right)-\Delta \rho^{S R O}\left(T_{i-1}\right)$

\footnotetext{
${ }^{2}$ Измеряется числом прыжков, приходящихся на 1 атом.
}

Nikolaev A. L. Short-range ordering in $\mathrm{Fe}-\mathrm{Cr}$ alloys irradiated with electrons at low temperatures and $\mathrm{Cr}$ solubility in $\alpha$-iron near $270 \mathrm{~K} / /$ Diagnostics, Resource and Mechanics of materials and structures. - 2021. - Iss. 6. - P. 6-18. - 
на $\Delta \rho_{0}^{d}$, которое пропорционально исходной концентрации дефектов $C_{0}^{d}$, получаемая разность $\Delta R^{S R O}\left(T_{i}\right)=R^{S R O}\left(T_{i}\right)-R^{S R O}\left(T_{i-1}\right)$ будет пропорциональна $N^{d}\left(T_{i}\right) / C_{0}^{d}$, т. е. среднему числу прыжков дефектов на данной ступени, приходящемуся на один исходный дефект. Так как в течение отжига сохраняется пропорциональность между текущей и исходной концентрациями дефектов, то величина $\Delta R^{S R O}\left(T_{i}\right)$ оказывается также пропорциональной среднему числу прыжков одного дефекта на данной ступени отжига ${ }^{3}$, т. е. $\Delta R^{S R O}\left(T_{i}\right) \sim n^{d}\left(T_{i}\right)$, где $n^{d}\left(T_{i}\right)$ есть среднее число прыжков одного дефекта при отжиге на данной ступени при $T_{i}$.

Известно, что в процессе отжига после низкотемпературного облучения дефекты взаимодействуют преимущественно друг с другом. Поэтому средний пробег (среднее число прыжков) одного дефекта на каждой ступени отжига определяется вероятностью его встречи с другими дефектами, которая обратно пропорциональна как текущей, так и исходной концентрациям дефектов. Для наших рассуждений важна обратная пропорциональность исходной концентрации дефектов, т.е. $\Delta R^{S R O}\left(T_{i}\right) \sim n^{d}\left(T_{i}\right) \sim 1 / C_{0}^{d}$. Отсюда следует, что абсолютная величина $\left|\Delta R^{S R O}\left(T_{i}\right)\right|$ уменьшается с увеличением исходной концентрации дефектов. Если рассматривать два подобных образца с разной концентрацией дефектов, высокой $(H)$ и низкой $(L)$, то всегда справедливо соотношение $\left|\Delta R_{L}^{S R O}\left(T_{i}\right)\right|>\left|\Delta R_{H}^{S R O}\left(T_{i}\right)\right|$.

Величина $R^{S R O}(T)$ после отжига на ступени при данной температуре $T$ есть сумма всех $\Delta R^{S R O}\left(T_{i}\right)$ на предшествующих ступенях отжига, включая $\Delta R^{S R O}(T)$ на последней ступени, т. е.

$$
R^{S R O}(T)=\sum_{i=1}^{T_{i}=T} \Delta R^{S R O}\left(T_{i}\right)
$$

и на каждой ступени $\left|\Delta R_{L}^{S R O}\left(T_{i}\right)\right|>\left|\Delta R_{H}^{S R O}\left(T_{i}\right)\right|$. В результате $\left|R_{L}^{S R O}(T)\right|>\left|R_{H}^{S R O}(T)\right|$ и разница между $\left|R_{L}^{S R O}(T)\right|$ и $\left|R_{H}^{S R O}(T)\right|$ растет с увеличением температуры.

На языке графиков увеличение разницы означает, что зависимости $R_{H}^{S R O}(T)$ и $R_{L}^{S R O}(T)$ начинают рост по абсолютной величине с нуля при температуре $T^{S R O}$ и далее расходятся с ростом температуры отжига. Если другие компоненты возврата ОЭС в $H$ - и $L$-образцах $R_{H}^{d}(T)$ и $R_{L}^{d}(T)$ различаются слабо, то расхождение $R_{H}^{S R O}(T)$ и $R_{L}^{S R O}(T)$ приводит к расхождению зависимостей $R_{H}(T)$ и $R_{L}(T)$. Таким образом, температура появление компоненты $R^{S R O}(T)$ в $R(T)$ легко определяется по началу расхождения зависимостей $R_{H}(T)$ и $R_{L}(T)$. Знак $R^{S R O}(T)$ без проблем определяется по порядку расхождения. Если $R^{S R O}(T)>0$, то кривая $R_{L}(T)$ проходит выше кривой $R_{H}(T)$. Если $R^{S R O}(T)<0$, то, соответственно, кривая $R_{L}(T)$ проходит ниже кривой $R_{H}(T)$.

Именно такую описанную выше картину расхождения кривых возврата ОЭС вследствие БУ, ускоренного дальней миграцией радиационных дефектов, наблюдали в облученном электронами аустенитном сплаве Fe-16Cr-20Ni [6] (рис. 1). Порядок расхождения соответствует $R^{S R O}(T)>0$, который увеличивается с ростом температуры отжига. Скорость роста

\footnotetext{
${ }^{3}$ Получается при нормировке общего числа прыжков всех дефектов на ступени на текущую концентрацию дефектов в начале этой ступени.
}

Nikolaev A. L. Short-range ordering in $\mathrm{Fe}-\mathrm{Cr}$ alloys irradiated with electrons at low temperatures and $\mathrm{Cr}$ solubility in $\alpha$-iron near $270 \mathrm{~K} / /$ Diagnostics, Resource and Mechanics of materials and structures. - 2021. - Iss. 6. - P. 6-18. DOI: $10.17804 / 2410-9908.2021 .6 .006-018$. 
$R^{S R O}(T)$ оказывается много больше скорости спада $R^{d}(T)$, в результате чего наблюдается рост $R(T)$ при отжиге после облучения. Далее мы попытаемся получить и использовать расходящиеся зависимости $R_{H}(T)$ и $R_{L}(T)$ в наших сплавах для решения поставленной задачи. Необходимо только иметь в виду, что в $\mathrm{Fe}-\mathrm{Cr} R^{S R O}(T)$ по отношению к $R^{d}(T)$ примерно в 30 раз меньше чем в $\mathrm{Fe}-16 \mathrm{Cr}-20 \mathrm{Ni}$ [9]. Поэтому закономерности расхождения $R(T)$, легко выявляемые в $\mathrm{Fe}-16 \mathrm{Cr}-20 \mathrm{Ni}$, могут потребовать определенных усилий для своего выявления в $\mathrm{Fe}-\mathrm{Cr}$.

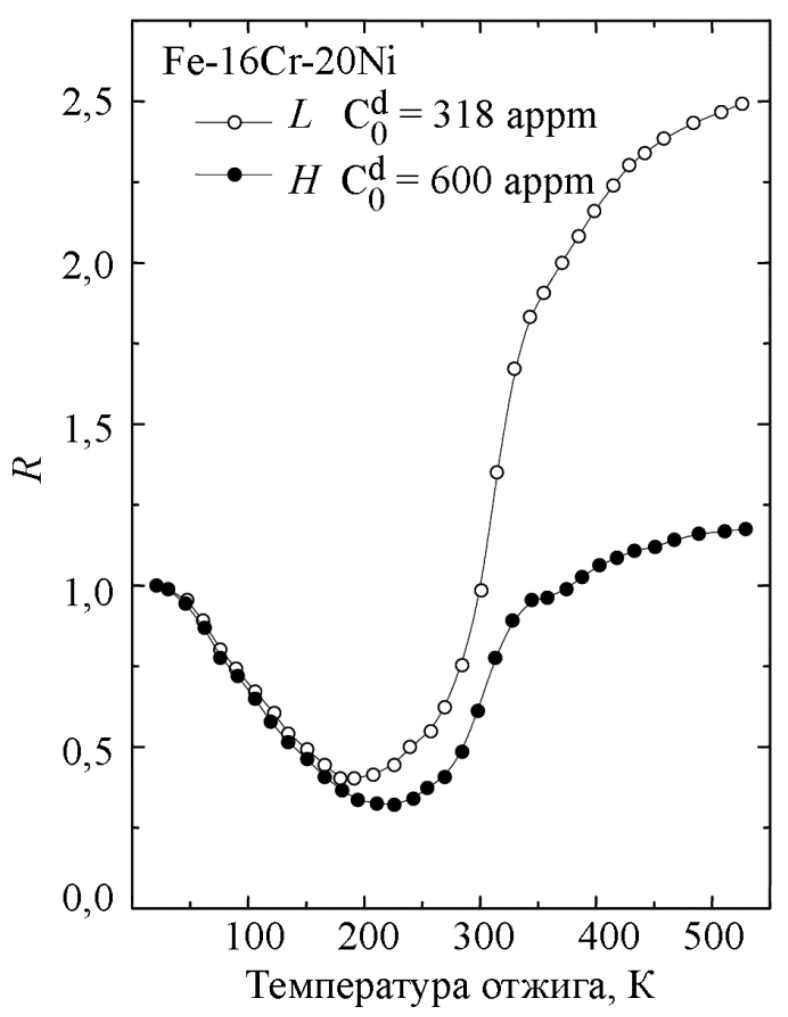

Рис. 1. Кривые восстановления ОЭС в образцах аустенитного сплава $\mathrm{Fe}-16 \mathrm{Cr}-20 \mathrm{Ni}$ после облучения 2,5 МэВ электронами при 21 К до различных концентраций радиационных дефектов согласно [5]

\section{3. Методика и характеристики образцов}

В настоящей работе использовали образцы четырех бинарных сплавов $\mathrm{Fe}-\mathrm{Cr}: \mathrm{Cr} 4$ (3,8 ат. \% Cr), $\mathrm{Cr} 6$ (6 ат. \% Cr), Cr9 (8,8 ат. \% Cr) и $\mathrm{Cr} 11$ (10,8 ат. \% Cr). Образцы готовили в дуговой печи переплавом на медном водоохлаждаемом поду спрессованных порошков высокочистого карбонильного железа и электролитического хрома. Концентрацию хрома задавали взвешиванием навесок с последующим контролем после переплава методом рентгеновского флуоресцентного анализа. Основные примеси - Ni (100 appm) и N (70 appm, в Cr6 $140 \mathrm{appm),} \mathrm{а} \mathrm{содержание} \mathrm{остальных} \mathrm{примесей} \mathrm{менее} 2$ appm. Детали приготовления и аттестации сплавов можно найти в [9].

Слитки раскатывали в фольги, из которых вырезали 4-контактные образцы для измерения ОЭС с размером измерительной части $4 \times 1,3 \times 0,06$ мм $^{3}$. Затем образцы отжигали в «безмасляном» (свободном от углеводородов) вакууме $10^{-4}$ Па при 1100 К в течение 4 ч. 
Измерения ОЭС проводили с помощью цифрового нановольтметра В2-39 (разрешение 1 нВ) и источника постоянного тока П-320 при измерительном токе 100 мА в жидком гелии. Относительная точность измерений составляла $\sim 10^{-5}$. Образцы облучали электронами с энергией 5 МэВ в проточном гелиевом криостате при температуре ниже 77 К. Для каждого сплава имелось по два образца ( $H$ - и $L$-), которые облучались до «высоких» $(2,2-2,4$ мкОм·см) и «низких» ( 0,4-0,6 мкОм·см) приростов ОЭС. После облучения образцы, не отогревая выше $77 \mathrm{~K}$, переносили в устройство для отжигов и измерения ОЭС в жидком гелии.

Далее проводили ступенчатые отжиги с шагом от 5 К до 10 К. Однако, дальнейший анализ показал, что $H$-образцы (т. е. которые облучались длительное время) в процессе облучения кратковременно (на несколько секунд или десятков секунд) перегревались до 125 К. Поэтому данные по возврату ОЭС для $H$ - и $L$-образцов обрабатывали немного по-разному. Данные для $L$-образцов обрабатывали обычным способом: текущие приросты ОЭС нормировали на приросты ОЭС после отжига при 90 К. По крайней мере до 170-180 К возврат ОЭС не зависит от прироста ОЭС. Поэтому возврат ОЭС в $H$-образцах приравнивали к возврату в $L$-образцах при 135 К и таким образом «сшивали» данные для $H$ - и $L$-образцов. Соответственно, данные по возврату ОЭС для $H$-образцов приводятся, начиная только со 135 К. Более подробное описание процедур облучения, измерения ОЭС и проведения ступенчатых отжигов можно найти в [9].

\section{4. Результаты и обсуждение}

На рис. 2 показаны зависимости восстановления ОЭС при отжиге после низкотемпературного облучения для четырех $L-H$ пар образцов сплавов $\mathrm{Cr} 4, \mathrm{Cr} 6, \mathrm{Cr} 9$ и $\mathrm{Cr} 11$. Видно, что, в целом, картина расхождения кривых заметно отличается от таковой в $\mathrm{Fe}-\mathrm{Cr}-\mathrm{Ni}$.

$\mathrm{B}$ сплавах 4Cr и 9Cr заметного расхождения не наблюдается вообще. В $\mathrm{Cr} 6$ и $\mathrm{Cr} 11$ наблюдается расхождение кривых возврата ОЭС с началом при 225 К и 195 К, соответственно, в общем, аналогичное расхождению кривых в сплаве $\mathrm{Fe}-16 \mathrm{Cr}-20 \mathrm{Ni}$. Порядок расхождения в Cr6 и Cr11 противоположный и соответствует БП в Cr6 и БР в Cr11. B Cr11 выше 245 К наблюдается падение ОЭС ниже исходного уровня. Падение ОЭС ниже исходного уровня является результатом $\Delta \rho_{C r 11}^{S R O}<0\left(R_{\mathrm{Cr} 11}^{\mathrm{SRO}}<0\right)$, т. е. БР, что согласуется с определением типа БУ, сделанным на основе порядка расходимости кривых восстановления ОЭС. Наблюдаемые типы БУ в Cr6 и Cr11 соответствуют данным работы [3] и подтверждают наличие концентрационного перехода от БП к БР, по крайней мере, до температур 225 К. Для того, чтобы завершить решение поставленной задачи необходимо определить тип БУ в сплаве Cr9. Так как $\mathrm{Cr} 9$ находится между $\mathrm{Cr} 6$ и $\mathrm{Cr} 11,225 \mathrm{~K}$ может рассматриваться как верхняя оценка для $T^{S R O}$.

Согласно данным ядерной гамма спектроскопии [4], в Cr4 наблюдается БП как при термическом отжиге вблизи $700 \mathrm{~K}$, так и при облучении электронами в температурном диапазоне 370-420 К. Поэтому, отсутствие расходимости кривых $R(T)$ в этом сплаве не связано с отсутствием процессов БУ, а скорее всего связано с тем, что расхождение кривых $R(T)$ маскируется другими процессами из-за малой величины $R^{\mathrm{SRO}}$ вследствие низкой концентрации хрома. Малую величину $R^{\mathrm{SRO}}$ можно также ожидать в $\mathrm{Cr} 9$ согласно данным ядерной гамма спектроскопии [4], вследствие близости сплава по концентрации к точке инверсии типа БУ. Поэтому, перед анализом данных по возврату ОЭС в $\mathrm{Cr} 9$, полезно проанализировать данные по возврату ОЭС в $\mathrm{Cr} 4$ на основе данных работ $[10,11]$, чтобы понять какие процессы могут маскировать расхождение кривых $R(T)$, обусловленное процессами БУ, и каким способом это расхождение можно выявить. Затем использовать полученные результаты для определения типа БУ в сплаве $\mathrm{Cr} 9$.

Nikolaev A. L. Short-range ordering in $\mathrm{Fe}-\mathrm{Cr}$ alloys irradiated with electrons at low temperatures and $\mathrm{Cr}$ solubility in $\alpha$-iron near $270 \mathrm{~K} / /$ Diagnostics, Resource and Mechanics of materials and structures. - 2021. - Iss. 6. - P. 6-18. - 


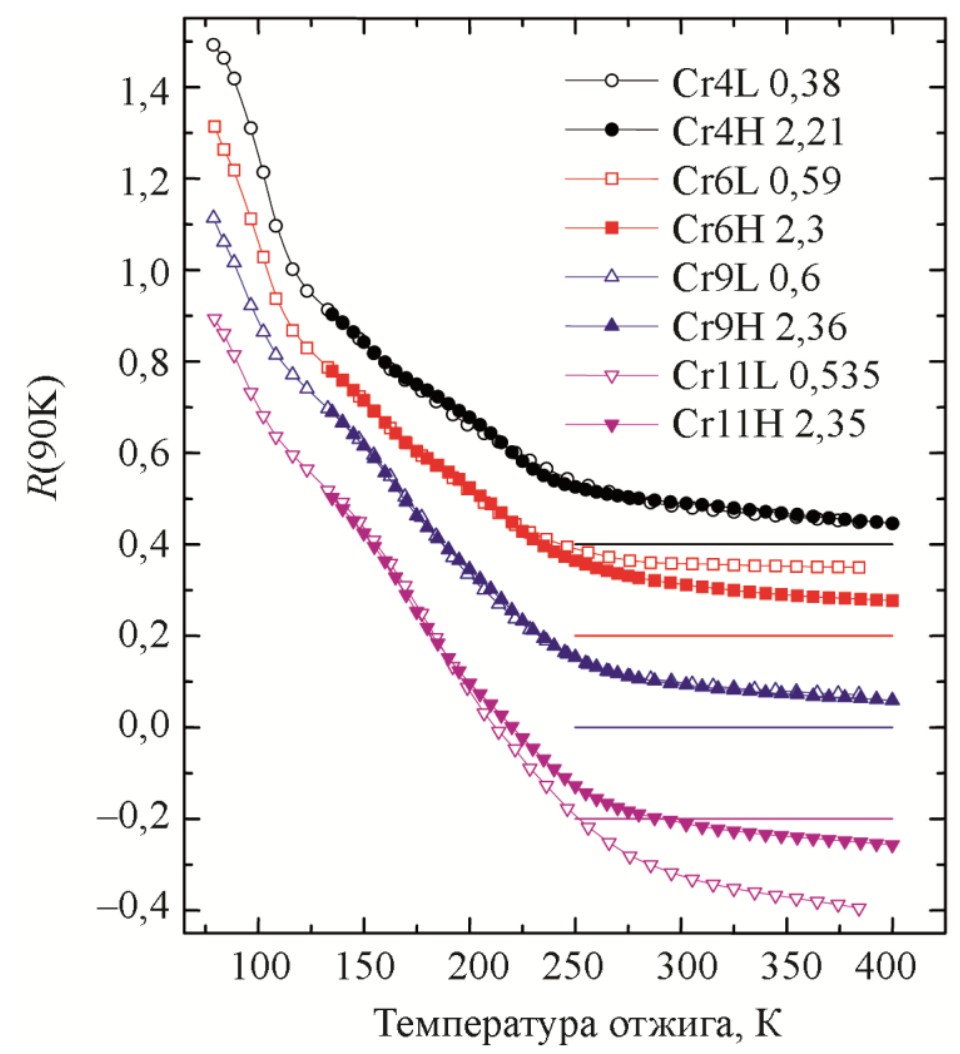

Рис. 2. Кривые восстановления ОЭС в сплавах $\mathrm{Fe}-\mathrm{Cr}$ после облучения 5 МэВ электронами при низких температурах до различных приростов ОЭС. Цифры в легенде указывают на величину прироста ОЭС в мкОм·см после отжига при 90 К. Кривые сдвинуты относительно друг друга по оси Ү на 0,2 и нулевой уровень для каждого сплава показан горизонтальной прямой соответствующего цвета

В системе $\mathrm{Fe}-\mathrm{Cr}$ наблюдаются значительные отклонения от правила Матиссена ${ }^{4}$ [12]. Это приводит к тому, что удельный вклад пар Френкеля в ОЭС становится зависимым от их концентрации. Эта зависимость приводит к расходимости между $L$ - и $H$-кривыми при температурах ниже начала дальней миграции дефектов ${ }^{5}$ и порядок этой расходимости оказывается обратным порядку расходимости, обусловленной БП. Примерно при 170 К начинается дальняя миграция вакансий и БУ по типу БП. Расходимость, вызванная отклонениями от правила Матиссена, начинает уменьшаться и при 195 К порядок расходимости меняется на противоположный, что соответствует БП, и такой порядок сохраняется до 230 К.

В сплавах $\mathrm{Fe}-\mathrm{Cr}$ существует явление аномального БР, индуцированного освобождением вакансий, захваченных на междоузельных примесях [11]. Этот тип БУ отличается от естественного БУ, которое нас интересует. В наших сплавах имеется примесь азота. Часть вакансий, которые начинают дальнюю миграцию выше $170 \mathrm{~K}$, захватывается на атомах азота. Освобождение захваченных вакансий происходит в температурном интервале 230-300 К [10]. Соответственно, в этом температурном интервале появляется индуцированное БР, влияние которого на ОЭС пересиливает влияние естественного БП. В результате, порядок расходимости при 230-300 К опять меняется. В результате такого чередования порядка расходимости суммарная расходимость при температурах выше $300 \mathrm{~K}$ оказывается малой и неразличимой

\footnotetext{
${ }^{4}$ Правило Матиссена утверждает, что вклады в электросопротивление от различных источников рассеяния электронов проводимости суммируются аддитивно.

${ }^{5}$ Небольшую расходимость между кривыми $R(T)$, также обусловленную отклонениями от правила Матиссена, можно наблюдать на рис. 1 в системе $\mathrm{Fe}-\mathrm{Cr}-\mathrm{Ni}$ при температурах ниже начала дальней миграции ( 170 К).
}

Nikolaev A. L. Short-range ordering in $\mathrm{Fe}-\mathrm{Cr}$ alloys irradiated with electrons at low temperatures and $\mathrm{Cr}$ solubility in $\alpha$-iron near $270 \mathrm{~K} / /$ Diagnostics, Resource and Mechanics of materials and structures. - 2021. - Iss. 6. - P. 6-18. DOI: 10.17804/2410-9908.2021.6.006-018. 
на рис. 2. Естественный способ избавиться от эффектов такого чередования - это анализировать процессы БУ выше 300 К, где отсутствуют вышеперечисленные процессы, маскирующие проявление естественного БУ в ОЭС.

Чтобы понять, как это можно сделать, обратимся к выражению (1), но суммирование начнем не с начала отжига, а с некоторой температуры $T^{+}$, т. е.

$$
R^{S R O}\left(T^{+}, T\right)=\sum_{T_{i}=T^{+}}^{T_{i}=T} \Delta R^{S R O}\left(T_{i}\right),
$$

и сравним значения такой «неполной» суммы в $L$ - и $H$-образцах, т.е. $R_{L}^{S R O}\left(T^{+}, T\right)$ и $R_{H}^{S R O}\left(T^{+}, T\right)$. Так же, как и в случае выражения (1) каждый член «неполной» суммы в $L$-образце будет больше по абсолютной величине соответствующего члена в $H$-образце. Поэтому, описываемые «неполной» суммой зависимости $R_{L}^{S R O}\left(T^{+}, T\right)$ и $R_{H}^{S R O}\left(T^{+}, T\right)$ будут расходиться также как $R_{H}^{S R O}(T)$ и $R_{L}^{S R O}(T)$, и порядок расхождения будет указывать на знак $R^{S R O}(T)$, т.е. тип БУ.

Чтобы использовать расхождение зависимостей $R_{L}^{S R O}\left(T^{+}, T\right)$ и $R_{H}^{S R O}\left(T^{+}, T\right)$ для определения типа БУ вблизи температуры $T^{+}$, необходимо использовать «неполный» возврат ОЭС $R_{L}\left(T^{+}, T\right)$ и $R_{H}\left(T^{+}, T\right)$ куда в качестве вклада, обусловленного БУ, будут входить $R_{L}^{S R O}\left(T^{+}, T\right)$ и $R_{H}^{S R O}\left(T^{+}, T\right)$.

«Неполную» сумму можно представить как разность $R^{S R O}\left(T^{+}, T\right)=R^{S R O}(T)-R^{S R O}\left(T^{+}\right)$, соответственно «неполный» возврат ОЭС должен выглядеть, как $R\left(T^{+}, T\right)=R(T)-R\left(T^{+}\right)$. В нашем случае $T^{+}=300 K$, поэтому следует анализировать расхождение зависимостей вида $R_{L}(T)-R_{L}(300 K)$ и $R_{H}(T)-R_{H}(300 K)$.

На рис. 3 а показаны «неполные» зависимости $R_{L}(T)-R_{L}(300 K)$ и $R_{H}(T)-R_{H}(300 K)$ для сплава $\mathrm{Cr} 4$, а также для сравнения такие же «неполные» зависимости для сплавов $\mathrm{Cr} 6$ и Cr11. Видно, что порядок расхождения «неполных» зависимостей для сплавов Cr6 и Cr11 такой же как и для «полных» зависимостей. Расхождение «неполных» зависимостей для $\mathrm{Cr} 4$ наблюдается выше 330 К и его порядок соответствует БП, что согласуется с типом БУ, обнаруженным при помощи ядерной гамма спектроскопии, после облучения электронами при близких (370 K) температурах [4]. Все это подтверждает возможность использования расходимости «неполных» зависимостей для определения типа БУ.

В сплаве $\mathrm{Cr} 9$ факторы, маскирующие расхождение кривых $R(T)$, отличаются от таких факторов в $\mathrm{Cr} 4$. Во-первых, индуцированное БР выражено намного слабее по сравнению с $\mathrm{Cr} 4$. Во-вторых, появляется дополнительный фактор. В целом, в $\mathrm{Fe}-\mathrm{Cr}$ зависимости $R_{H}^{d}(T)$ и $R_{L}^{d}(T)$ различаются слабо, за исключением одного температурного интервала. В этих сплавах дальняя миграция дефектов начинается с миграции свободных вакансий. Соответствующая этой миграции стадия возврата ОЭС располагается в температурном интервале 170-240 К [9]. Но известно, что положение стадии миграции свободных дефектов сдвигается с изменением концентрации дефектов. Этот сдвиг приводит к появлению расхождения между $R_{H}^{d}(T)$ и $R_{L}^{d}(T)$ в температурном интервале стадии миграции вакансий, и может мешать увидеть расхождение, обусловленное БУ при 170-240 К. Этот фактор пренебрежимо мал в Cr4, но достаточно велик в Cr9 и препятствует определению типа БУ ниже 240 К. Поэтому в Cr9 следует анализировать расхождение «неполных» зависимостей выше $250 \mathrm{~K}$. 


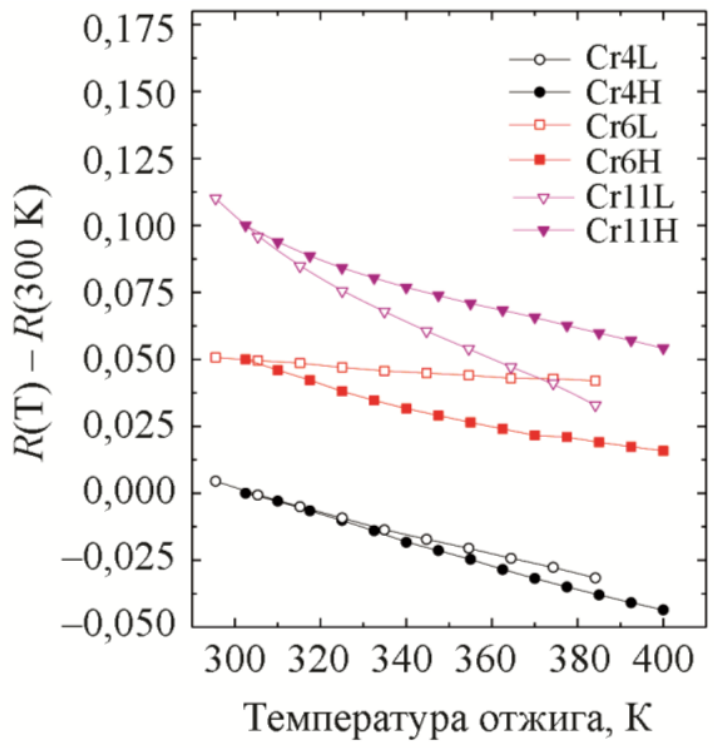

$a$

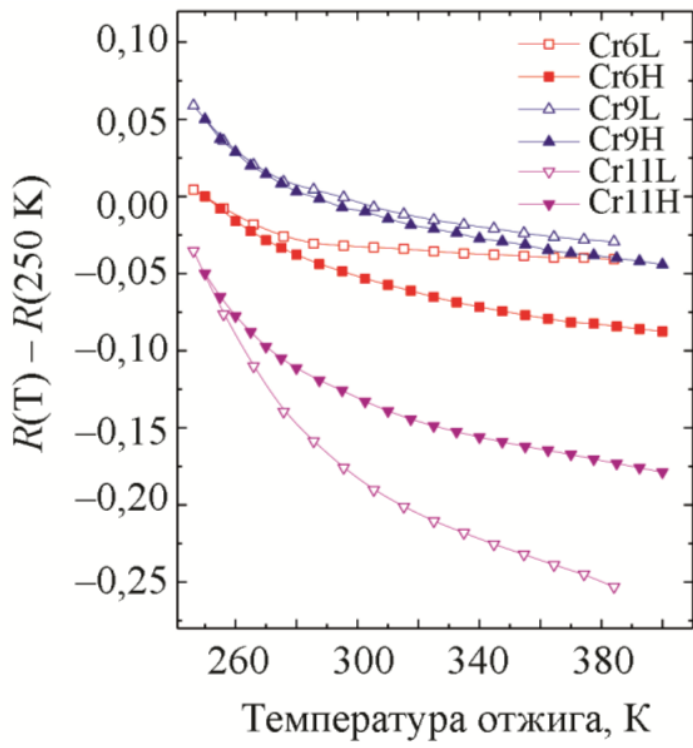

$\sigma$

Рис. 3. «Неполные» зависимости восстановления ОЭС для образцов сплавов $\mathrm{Fe}-\mathrm{Cr}$ (рис. 2) при различных значениях параметра $T^{+}: a-T^{+}=300 \mathrm{~K} ; \sigma-T^{+}=250 \mathrm{~K}$. Кривые сдвинуты относительно друг друга по оси $Y$ на 0,05

На рис. 3 б показаны «неполные» зависимости $R_{L}(T)-R_{L}(250 K)$ и $R_{H}(T)-R_{H}(250 K)$ для сплава $\mathrm{Cr} 9$ и такие же «неполные» зависимости для сплавов $\mathrm{Cr} 6$ и $\mathrm{Cr} 11$ для сравнения. Видно, что в Cr9 выше 270 К наблюдается систематическое расхождение «неполных» зависимостей и порядок расхождения указывает на БП. Порядок расхождения в сплавах Cr6 и Cr11 соответствует БП и БР соответственно. Таким образом, при температурах выше 270 К концентрация хрома в сплавах $\mathrm{Cr} 6$ и $\mathrm{Cr} 9$ находится ниже предела растворимости.

Хотя согласно рентгеновскому флюоресцентному анализу содержание хрома в $\mathrm{Cr} 9$ составляет 8,8 ат. \%, часть хрома (до 0,4 ат. \%) может находится в окислах (рентгеновский флюоресцентный анализ не различает хром в твердом растворе и окислах). Это связано с тем, что исходное карбонильное железо содержит до 1 ат. \% адсорбированного кислорода [13] и в присутствии этого кислорода часть хрома может «выгорать». Поэтому нижняя оценка концентрации хрома в сплаве $\mathrm{Cr} 9$ равняется 8,4 ат. \% Cr. Верхняя оценка предела растворимости согласно данным $[1,2]$ составляет 9,2 ат. \% Cr. Соответственно, доверительный интервал для предела растворимости при температурах чуть выше 270 К равен 8,8 $\pm 0,4$ ат. \% Cr.

\section{5. Выводы}

Проанализированы данные по возврату ОЭС в сплавах $\mathrm{Cr} 4, \mathrm{Cr} 6, \mathrm{Cr} 9$ и $\mathrm{Cr} 11$ после низкотемпературного облучения 5 МэВ электронами с точки зрения наблюдения процессов БУ, ускоренных дальней миграцией радиационных дефектов.

1. Показано, что прямое наблюдение расхождения кривых возврата ОЭС для образцов с разной начальной концентрацией дефектов, указывающее на процессы БУ, возможно не во всех сплавах. Расхождение наблюдается в Cr6 и Cr11, начиная с 225 К и 195 K, соответственно, и указывает на БП в Сr6 и БР в Cr11. Отсюда следует, что переход по типу БУ от БП к БР наличествует в интервале концентраций от 6 до 11 ат. \% Сr вплоть до температур 225 К.

2. Показано, что для идентификации процесса БУ и определения его типа в сплавах $\mathrm{Cr} 4$ и $\mathrm{Cr} 9$ можно использовать расхождение «неполных» кривых возврата ОЭС. Расхождение «неполных» кривых возврата ОЭС, обусловленное БУ, удается наблюдать в $\mathrm{Cr} 4$ и $\mathrm{Cr} 9$ начиная с $330 \mathrm{~K}$ и 270 К, соответственно, и порядок расхождения указывает на БП в обоих сплавах. 
3. Идентификация БП в сплаве $\mathrm{Cr} 9$ при температурах выше 270 К позволяет определить доверительный интервал для значений предела растворимости хрома в $\alpha$-железе для

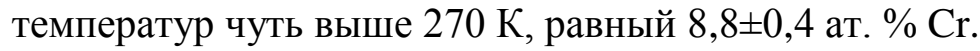

\section{Благодарности}

Работа выполнена в рамках государственного задания МИНОБРНАУКИ России (тема «Функиия», № AAAA-A19-119012990095-0).

\section{Литература}

1. Bachhav Mukesh, Odette G. Robert, Marquis Emmanuelle A. $\alpha^{\prime}$ precipitation in neutron-irradiated Fe-Cr alloys // Scripta Materialia - 2014. - Vol. 74. - P. 48-51. DOI: $10.1016 /$ j.scriptamat.2013.10.001.

2. Kinetics of $\alpha^{\prime}$ precipitation in an electron-irradiated $\mathrm{Fe} 15 \mathrm{Cr}$ alloy / O. Tissot, C. Pareige, E. Meslin, B. Decamps, J. Henry // Scripta Materialia. - 2016. - Vol. 122. - P. 31-35. DOI: 10.1016/J.SCRIPTAMAT.2016.05.021.

3. Mirebeau I., Hennion M., Parette G. First measurement of short-range-order inversion as a function of concentration in a transition alloy // Phys. Rev. Letters -1984. - Vol. 53, iss. 7. P. 687-690. - DOI: 10.1103/PHYSREVLETT.53.687.

4. Filippova N. P., Shabashov V. A., Nikolaev A. L. Mössbauer Study of IrradiationAccelerated Short-Range Ordering in Binary Fe-Cr Alloys // Physics of Metals and Metallography. 2000. - Vol. 90, No. 2 - P. 145-152.

5. Dimitrov C., Dimitrov O. Composition dependence of defect properties in electronirradiated Fe-Cr-Ni solid solutions // J. Phys. F: Met. Phys. - 1984. - Vol. 14 - P. 793- 811. DOI: 10.1088/0305-4608/14/4/005.

6. Vaessen P., Lengeler B., Shilling W. Recovery of electrical resistivity in electronirradiated concentrated silver-zinc alloys // Rad. Effects - 1984. - Vol. 81. - P. 277-292. DOI: $10.1080 / 00337578408206075$.

7. Ruban A. V., Korzhavyi P. A., Johansson B. First-principles theory of magnetically driven anomalous ordering in bcc Fe-Cr alloys // Phys. Rev. B - 2008. -Vol. 77. iss 9. - P. 094436. DOI: 10.1103/PHYSREVB.77.094436.

8. Investigation of the kinetics of short-range order formation and quenched-in vacancy annihilation in Au-15 at. \% Ag by resistivity measurements / W. Kohl, R. Scheffel, H. Heidsiek, K. Lucke // Acta metall. - 1983. - Vol. 31, No. 11. - P. 1895-1908. - DOI: 10.1016/0001-6160(83)90135-9.

9. Nikolaev A. L. Specificity of stage III in electron-irradiated Fe-Cr alloys // Phil. Mag. 2007. - Vol. 87, No. 31. - P. 4847-4874. - DOI: 10.1080/14786430701468977.

10. Nikolaev A. L. Difference approach to the analysis of resistivity recovery data for irradiated short-range ordered alloys // Phil. Mag. - 2009. - Vol. 89, No. 12. - P. 1017-1033. DOI: $10.1080 / 14786430902835651$.

11. Nikolaev A. L. Recovery of electrical resistivity, short-range order formation and migration of defects in electron-irradiated Fe-4Cr alloy doped with carbon // Phil. Mag. - 2011. - Vol. 91, No. 6. - P. 879-898. - DOI: 10.1080/14786435.2010.534740.

12. A study of irradiated FeCr alloys: deviations from Matthiessen's rule and interstitial migration / F. Maury, P. Lucasson, A. Lucasson, F. Faudot, J. Bigot // J. Phys. F: Met. Phys. 1987. - Vol. 17. - P. 1143-1165. - DOI: 10.1088/0305-4608/17/5/014.

13. Nikolaev A. L. Stage I of recovery in $5 \mathrm{MeV}$ electron-irradiated iron and iron-chromium alloys: the effect of small cascades, migration of di-interstitials and mixed dumbbells // J. Phys.: Condens. Matter. - 1999. - Vol. 11. - P. 8933-8644. - DOI: 10.1088/0953-8984/11/44/302. 